\title{
Cellular Differentiation-Based Service Adaptation
}

\author{
Ichiro Satoh \\ National Institute of Informatics \\ 2-1-2 Hitotsubashi, Chiyoda-ku, Tokyo 101-8430, Japan \\ ichiro@nii.ac.jp
}

\begin{abstract}
This paper proposes an approach to adapting services in a distributed system whose computational resources are dynamically changed. It supports the notions of cellular differentiation and dedifferentiation. When a service delegates a function to another component coordinating with it, if the former has the function, this function becomes less-developed and the latter's function becomes well-developed. When some differentiated services are not available, it enables remaining services to automatically support the functions provided from the unavailable services. The approach was constructed as a middleware system and allowed us to define agents as Java objects. We present several evaluations of the framework in a distributed system.
\end{abstract}

\section{Introduction}

Cellular differentiation is the mechanism by which cells in a multicellular organism become specialized to perform specific functions in a variety of tissues and organs. Different kinds of cell behaviors can be observed during embryogenesis: cells double, change in shape, and attach at and migrate to various sites within the embryo. This paper introduces the notion of cellular differentiation into distributed systems as an adaptive approach. Distributed systems tend to be dynamic by nature because units, e.g., computers and networks, may fail and new units may have to be added to include new resources, applications, or users. Furthermore, such units tend to be heterogeneous and have limited computational resources. A federation of services running on multiple computers whose computational resources are different is needed to support functions beyond the capabilities of individual computers.

Our approach involves service matching as the service differentiation factors in services. When a service delegates a function to another service, if the former has the function, its function becomes less-developed and the latter's function becomes welldeveloped. As a result, differentiated services become specialized or degenerated according to demands from other services. Furthermore, the approach also supports the concept of dedifferentiation for managing contingencies, e.g., network partitioning and system or service failures, where dedifferentiation is the process of reverting partially or terminally differentiated cells to an earlier developmental stage.

\section{Background}

Service composition involves the development of customized services often by discovering, integrating, and executing existing services. Most existing work on service 
composition has been designed to support coordination/mashups between services rather than adapting service themselves. Several researchers have explored genetic computation/programming [5] and swarm intelligence [2]. Such approaches have often been too diverse, but real systems may have no chance of ascertaining the fitness of randomly generated parameters or programs, and many agents because they have an effect on the real world and no surplus computational resources. There have been several attempts to support software adaptation in the literature on adaptive computing. Blair et al. [1] tried to introduce self-awareness and self-healing into a CORBA-compatible Object Request Broker (ORB). Their system had a meta-level architecture with the ability of dynamically binding CORBA objects. Several researchers had proposed bio-inspired middleware for dynamic and large-scale networks [7]. Although they introduced the notion of energy into distributed systems and have enabled agents to be replicated, moved, and deleted according to the number of service requests, they have had no mechanism for adapting agents' behaviors unlike ours.

\section{Basic Approach}

Our approach assumes that each service is defined as an autonomous software component, called an agent, that is like a cell and consists of one or more functions that can be invoked by itself or other agents. The approach introduces the undertaking/delegation of behaviors in agents from other agents as a differentiation factor. Behaviors in an agent, which are delegated from other agents more frequently in a cell, are well developed, whereas other behaviors, which are delegated from other agents less frequently, in the cell are less developed. Finally, the agent only provides the former behaviors and delegates the latter behaviors to other agents.
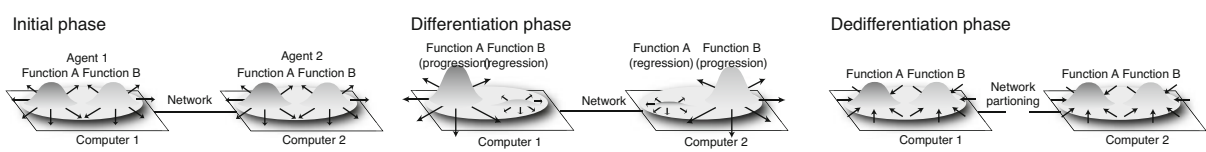

Fig. 1. Differentiation mechanism for software configuration

Differentiation: When dictyostelium discoideum cells aggregate, they can be differentiated into two types: prespore cells and prestalk cells. Each cell tries to become a prespore cell and periodically secretes cAMP to other cells. If a cell can receive more than a specified amount of cAMP from other cells, it can become a prespore cell. There are three rules. 1) cAMP chemotaxically leads other cells to prestalk cells. 2) A cell that is becoming a prespore cell can secrete a large amount of cAMP to other cells. 3) When a cell receives more cAMP from other cells, it can secrete less cAMP to other cells (Fig.1).

Each agent has one or more functions with weights, where each weight corresponds to the amount of cAMP and indicates the superiority of its function. Each agent initially intends to improve all its functions and periodically multicasts restraining messages 
to other agents federated with it instead of cAMP. The messages lead other agents to degenerate their functions specified in the messages and to decrease the superiority of these functions. As a result, agents complement others in the sense that each agent can provide some functions to other agents and delegate other functions to other agents that can also provide the functions.

Dedifferentiation: Agents may lose their functions due to differentiation as well as being busy or having failed. The approach also offers a mechanism for recovering from such problems based on dedifferentiation, which is a mechanism for regressing specialized cells to simpler, more embryonic, unspecialized forms. If there are no other agents that are sending restraining messages to an agent, the agent can perform its dedifferentiation process and strengthen their less-developed or inactive functions again.

\section{Design and Implementation}

The whole system consists of two parts: runtime systems and agents. The former is a middleware system for running at computers and the latter is a self-contained and autonomous entity and corresponds to a service and consists of more than one function, called the behavior part, and its state, called the body part, with information for differentiation, called the attribute part. The first part defines more than one application-specific behavior, which is a general/practical-purpose program defined in JavaBean-compatible Java objects. The second is responsible for maintaining program variables shared by its behaviors parts. When it receives a request message from an external system or other agents, it dispatches the message to the behavior part that can handle the message. The third maintains descriptive information with regard to the agent, including its own identifier in its two key-value databases for maintaining the weights of its own behaviors and for recording information on the behaviors that other agents can provide. Note that we never expect that the latter is complete. In fact, the mechanism can still work, even if it is not complete.

\subsection{Differentiation}

Each agent ( $k$-th) assigns its own maximum to the total of the weights of all its behaviors. The agent has behaviors $b_{1}^{k}, \ldots, b_{n}^{k}$ and $w_{i}^{k}$ is the weight of behavior $b_{i}^{k}$. The $W_{i}^{k}$ is the maximum of the weight of behavior $b_{i}^{k}$. The maximum total of the weights of its behaviors in the $k$-th agent must be less than $W^{k}$. $\left(W^{k} \geq \sum_{i=1}^{n} w_{i}^{k}\right)$, where $w_{j}^{k}-1$ is 0 if $w_{j}^{k}$ is 0 . The $W^{k}$ may depend on agents. Our mechanism consists of two phases. The first-step phase involves the progression of behaviors in four steps.

Step 1: When an agent ( $k$-th agent) receives a request message from another agent, it selects the behavior $\left(b_{i}^{k}\right)$ that can handle the message from its behavior part and dispatches the message to the selected behavior (Figure2(a)).

Step 2: It executes the behavior $\left(b_{i}^{k}\right)$ and returns the result.

Step 3: It increases the weight $w_{i}^{k}$ of the behavior.

Step 4: It multicasts a restraining message with the signature of the behavior, its identifier $(k)$, and the behavior's weight $\left(w_{i}^{k}\right)$ to other agents (Figure $2(\mathrm{~b})$ ). 


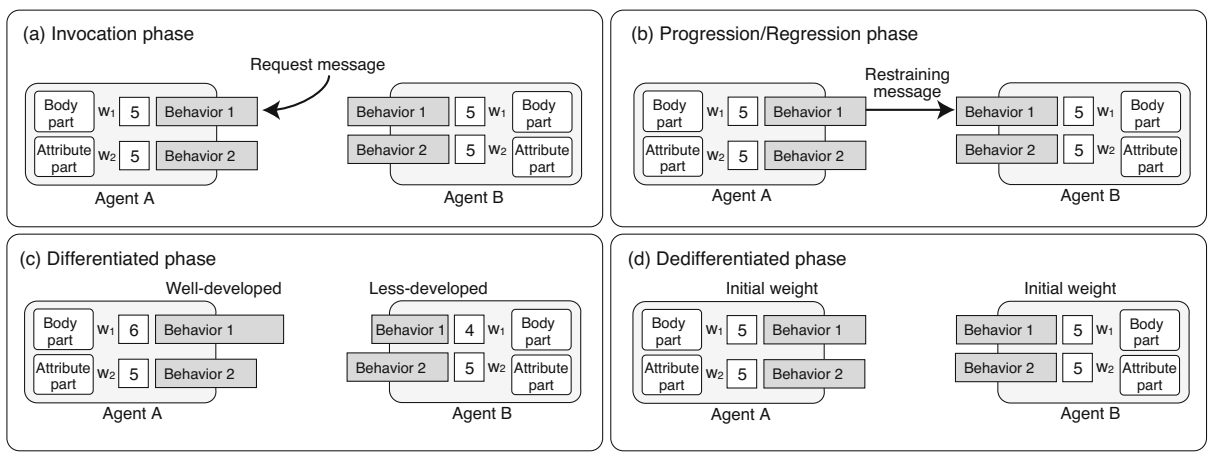

Fig. 2. Differentiation mechanism for agent

Note that when behaviors are internally invoked by their agents, their weights are not increased. The key idea behind this approach is to distinguish between internal and external requests. If the total weights of the agent's behaviors, $\sum w_{i}^{k}$, is equal to their maximal total weight $W^{k}$, it decreases one of the minimal (and positive) weights $\left(w_{j}^{k}\right.$ is replaced by $w_{j}^{k}-1$ where $w_{j}^{k}=\min \left(w_{1}^{k}, \ldots, w_{n}^{k}\right)$ and $\left.w_{j}^{k} \geq 0\right)$. The above phase corresponds to the degeneration of agents. Restraining messages correspond to cAMP in differentiation. When the runtime system multicasts information about the signature of a behavior in restraining messages, the signature is encoded into a hash code by using Java's serial versioning mechanism and transmitted as code. The second-step phase supports the retrogression of behaviors in three steps.

Step 1: When an agent ( $k$-th agent) receives a restraining message with regard to $b_{i}^{j}$ from another agent $\left(j\right.$-th), it looks for the behaviors $\left(b_{m}^{k}, \ldots b_{l}^{k}\right)$ that can satisfy the signature specified in the received message.

Step 2: If it has such behaviors, it decreases their weights $\left(w_{m}^{k}, \ldots w_{l}^{k}\right)$ in its first database and updates the weight $\left(w_{i}^{j}\right)$ in its second database (Figure2(c)).

Step 3: If the weights $\left(w_{m}^{k}, \ldots, w_{l}^{k}\right)$ are under a specified value, e.g., 0 , the behaviors $\left(b_{m}^{k}, \ldots b_{l}^{k}\right)$ are inactivated.

\subsection{Service Matching}

When an agent wants to execute a behavior, it needs to select one of the behaviors, even if it has the behavior, according to the values of their weights. This involves three steps.

Step 1: When an agent ( $k$-th agent) wants to execute behavior $b_{i}$, it looks up the weight $\left(w_{i}^{k}\right)$ of the same or compatible behavior from its first database and the weights $\left(w_{i}^{j}, \ldots, w_{i}^{m}\right)$ of such behaviors $\left(b_{i}^{j}, \ldots, b_{i}^{m}\right)$ from the second database 1

Step 2: If multiple agents, including itself, can provide the wanted behavior, it selects one of the agents according to selection function $\phi^{k}$, which maps from $w_{i}^{k}$ and $w_{i}^{j}, \ldots, w_{i}^{m}$ to $b_{i}^{l}$, where $l$ is $k$ or $j, \ldots, m$.

Step 3: It delegates the selected agent to execute the behavior and waits for the result from the agent.

\footnotetext{
${ }^{1}$ The agent $(k$-th) may have more than one same or compatible behavior.
} 
The approach permits agents to use their own evaluation functions, $\phi$, because the selection of behaviors often depends on their applications. For example, one of the simplest evaluation functions makes the agent that wants to execute a behavior select a behavior whose weight has the highest value and whose signature matches the wanted behavior if its first and second databases recognizes one or more agents that provide the same behavior, including itself. There is no universal selection function for mapping from behaviors' weights to at most one appropriate behavior like that in a variety of creatures. Therefore, the approach is open to defining functions by over-writing Java classes for a selection function.

\subsection{Dedifferentiation}

We need a mechanism for detecting failures in networking, remote computers, and other agents. To do this, each agent ( $j$-th) periodically multicasts messages, called heartbeat messages, for behavior $\left(b_{i}^{j}\right)$, which is still activated with its identifier $(j)$. This involves two cases.

Case 1: When an agent ( $k$-th) receives a heartbeat message with regard to behavior $\left(b_{i}^{j}\right)$ from another agent $\left(j\right.$-th), it keeps the weight $\left(w_{i}^{j}\right)$ of the behavior $\left(b_{i}^{j}\right)$ in its second database.

Case 2: When an agent ( $k$-th) does not receive any heartbeat messages with regard to behavior $\left(b_{i}^{j}\right)$ from another agent $(j$-th) for a specified time, it automatically decreases the weight $\left(w_{i}^{j}\right)$ of the behavior $\left(b_{i}^{j}\right)$ in its second database, and resets the weight $\left(w_{i}^{k}\right)$ of the behavior $\left(b_{i}^{k}\right)$ to the initial value or increases the weight $\left(w_{i}^{k}\right)$ in its first database (Figure2(d)).

The weights of behaviors provided by other agents are automatically decreased without any heartbeat messages from the agents. Therefore, when an agent terminates or fails, other agents decrease the weights of the behaviors provided from the agent and if they have the same or compatible behaviors, they can then activate the behaviors, which may be inactivated. After a request message is sent to another agent, if the agent waits for the result to arrive for longer than a specified time, it selects one of the agents that can handle the message from its first and second databases and requests the selected agent. If there are no agents that can provide the behavior that can handle the behavior quickly, it promotes other agents that have the behavior in less-developed form (and itself if it has the behavior).

\subsection{Current Status}

Each runtime system is constructed as a middleware system that enables agents to duplicate themselves and migrate to other computers by using mobile agent technology [6]. It is responsible for executing agents and exchanging messages in runtime systems on other computers through a network. Restraining and heartbeat messages are multicasted as UDP packets, which may be unreliable. When a runtime system is (re)connected to a network, it multicasts heartbeat messages to other runtime systems to advertise itself, including its network address through UDP multicasts. Request and reply messages are implemented through TCP sessions. 
Although the current implementation was not constructed for performance, we evaluated that of several basic operations in a distributed system where eight computers (Intel Core 2 Duo $1.83 \mathrm{GHz}$ with MacOS X 10.6 and J2SE version 6) were connected through a Giga Ethernet. The cost of transmitting a heartbeat or restraining message through UDP multicasting was $11 \mathrm{~ms}$. The cost of transmitting a request message between two computers was $22 \mathrm{~ms}$ through TCP2

\section{Evaluation}

This experiment was aimed at evaluating the basic performance of adaptation, where one or more simple agents at computers and each agent issued heartbeat messages every $100 \mathrm{~ms}$. Figure 3 shows the evaluation of differentiation. Each agent had three behaviors, called A, B, and $\mathrm{C}$. The A behavior periodically issued messages to invoke its $\mathrm{B}$ and $\mathrm{C}$ behaviors or those of other agents every $200 \mathrm{~ms}$ and the $\mathrm{B}$ and $\mathrm{C}$ behaviors were null behaviors. Each agent that wanted to execute a behavior, i.e., B or C, selected a behavior whose weight had the highest value if its database recognized one or more agents that provided the same or compatible behavior, including itself. When it invoked behavior $\mathrm{B}$ or $\mathrm{C}$ and the weights of its and others behaviors were the same, it randomly selected one of the behaviors. The weights of the $\mathrm{B}$ and $\mathrm{C}$ behaviors of each agent in this experiment would initially be five and the maximum weight of each behavior and the total maximum of weights would be ten.

a) Differentiation in two agents

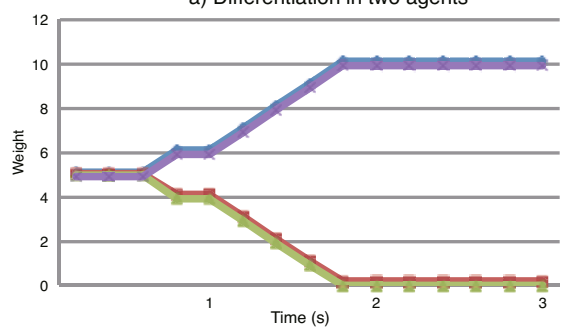

c) Differentiation in eight agents

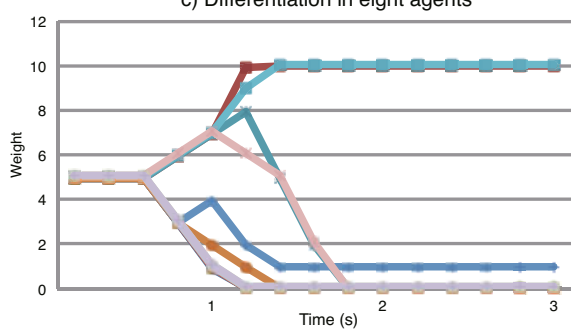

b) Differentiation in four agents

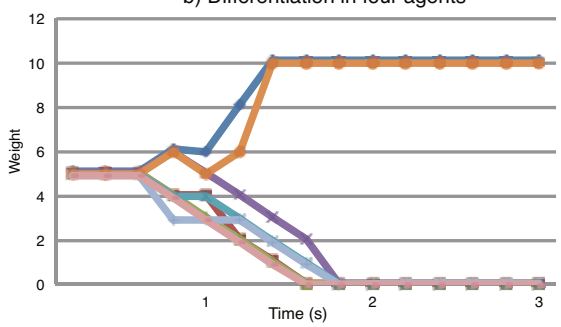

Fig. 3. Degree of progress in differentiation-based adaptation

\footnotetext{
${ }^{2}$ These costs were estimated from measurements of round-trip times between computers.
} 
Figure 3 presents the results we obtained from the experiment. Both diagrams have a timeline on the $\mathrm{x}$-axis and the weights of behavior $\mathrm{B}$ in each agent on the $\mathrm{y}$-axis. Figure 3 (a) details the results obtained from our differentiation between two agents. Their weights were not initially varied and then they forked into progression and regression sides. Figure 3 (b) shows the detailed results of our differentiation between four agents and Figure 3 (c) shows those of that between eight agents. The results in (b) and (c) fluctuated more and then converged faster than those in (a) because the weights of behaviors in four agents were increased or decreased more than those in two agents. Although the time of differentiation depended on the period behaviors were invoked it was independent of the number of agents. This is important to prove that this approach is scalable.

Figure 4 shows the evaluation of dedifferentiation against failures in a distributed system. We assumed in the following experiment that three differentiated agents would be running on different computers and each agent had four behaviors, called A, B, C, and D, where the A behavior invoked other behaviors every $200 \mathrm{~ms}$. The maximum for each behavior was ten and the agents' total maximum of weights was twenty. The initial weights of their behaviors $\left(w_{B}^{i}, w_{C}^{i}, w_{D}^{i}\right)$ in the $i$-th agent were $(10,0,0)$ in the first, $(0,10,0)$ in the second, and $(0,0,10)$ in the third.
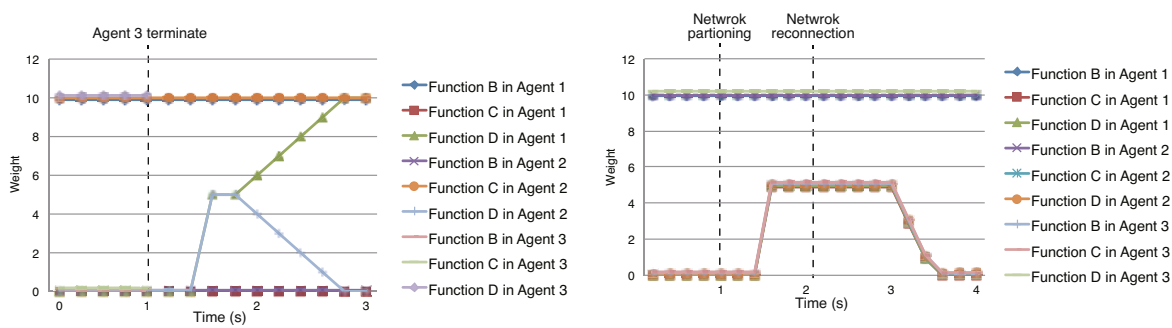

Fig. 4. Degree of progress in adaptation to failed agent

Figure 4 shows how remaining agents adapt to the termination of an agent. The third agent was terminated one second later. The remaining agents, i.e., the first and second agents, could not invoke the behavior, i.e., D, provided by it, and they dedifferentiated the behavior inside themselves.

\section{Application}

Here, we present a practical application with this approach to illustrate the utility of our (de)differentiation for service deployment and composition in a disaggregated computing setting. This application was inspired by the concept of disaggregated computing, which is an approach to dynamically composing devices, e.g., displays, keyboard, and mice that are not attached to the same computer, into a virtual computer among distributed computers in an ambient computing environment [3].

Our application is constructed as a single drawing service consisting of model, view, and control behaviors like a model-view-control (MVC) pattern. The first manages and stores drawing data and should be executed on a computer equipped with a powerful processor and a lot of memory. The second part displays drawing data on the screen 
of its current host and should be deployed at computers equipped with large screens. The third part forwards drawing data from the pointing device, e.g., touch panel, of its current computer to the first behavior.

The service is initially deployed at a server, which lacks displays and storage. When the server is connected to a network, its runtime system discovers a computer equipped with a pointing device and a large display, e.g., a smart TV; the agent makes a clone of it with its behaviors and deploys the clone agent at the smart TV. The original agent, which is running on the server, decreases the weights of its behaviors corresponding to the view and control parts and the clone agent, which is running on the smart TV, decreases the weight of its behavior corresponding to the model part. This is because the server has no display or pointing device and the smart TV had no storage device. Therefore, each of the agents delegates the behaviors that its computer does not support to another agent according to the capabilities of their current computers. When a user disconnects the server from the network, the agent running on the server dedifferentiates itself, because it lacks co-partners, and it delegates the behaviors corresponding to the view and control parts.

\section{Conclusion}

This paper proposed a approach to adapting services on distributed systems, in particular ambient computing environments. It is unique to other existing software adaptations in introducing the notions of differentiation and dedifferentiation in cellular slime molds, e.g., dictyostelium discoideum, into software agents. When an agent delegates a behavior to another agent, if the former has the behavior, its behavior becomes lessdeveloped and the latter's behavior becomes well-developed. The approach was constructed as a middleware system on real distributed systems instead of any simulationbased systems.

\section{References}

1. Blair, G.S., Coulson, G., Blair, L., Duran-Limon, H., Grace, P., Moreira, R., Parlavantzas, N.: Reflection, self-awareness and self-healing in OpenORB. In: Proceedings of 1st Workshop on Self-healing systems (WOSS 2002), pp. 9-14. ACM Press (2002)

2. Bonabeau, E., Dorigo, M., Theraulaz, G.: Swarm Intelligence: From Natural to Artificial Systems. Oxford University Press (1999)

3. Brumitt, B.L., Meyers, B., Krumm, J., Kern, A., Shafer, S.: EasyLiving: Technologies for Intelligent Environments. In: Proceedings of International Symposium on Handheld and Ubiquitous Computing, pp. 12-27 (2000)

4. Georgiadis, I., Magee, J., Kramer, J.: Self-Organising Software Architectures for Distributed Systems. In: Proceedings of 1st Workshop on Self-healing systems (WOSS 2002), pp. 33-38. ACM Press (2002)

5. Koza, J.R.: Genetic Programming: On the Programming of Computers by Means of Natural Selection. MIT Press (1992)

6. Satoh, I.: Handbook of Ambient Intelligence and Smart Environments, pp. 771-791. Springer, Heidelberg (2010)

7. Suda, T., Suzuki, J.: A Middleware Platform for a Biologically-inspired Network Architecture Supporting Autonomous and Adaptive Applications. IEEE Journal on Selected Areas in Communications 23(2), 249-260 (2005) 